\title{
Effects of Pathological Conditions on Ocular Pharmacokinetics of Antimicrobial Drugs
}

\author{
Kayoko UedA, ${ }^{* a, b}$ Akira Ohtori, ${ }^{a}$ and Kakuji Tojo ${ }^{b}$ \\ ${ }^{a}$ Research Laboratories Senju Pharmaceutical Co., Ltd.; 1-5-4 Murotani, Nishi-ku, Kobe, Hyogo 651-2241, Japan: and \\ ${ }^{b}$ Faculty of Computer Science and Systems Engineering, Kyushu Institute of Technology; 680-4 Kawazu, Iizuka, Fukuoka \\ 820-8502, Japan. Received December 1, 2009; accepted July 24, 2010; published online July 27, 2010
}

A diffusion model of ocular pharmacokinetics was used to estimate the effects of pathological conditions on ocular pharmacokinetics. In vivo rabbit data after topical instillation of ciprofloxacin and ofloxacin were compared with the simulated concentrations in the aqueous and vitreous humors. The barrier capacity of the surrounding membranes such as the retina/choroid/sclera (RCS) membrane and the cornea was characterized by dimensionless Sherwood number derived by the pseudo-steady state approach (PSSA). We assumed the barrier capacity decreased by inflammation; when the barrier capacity of the RCS membrane and the cornea was assumed to be one-tenth for the RCS membrane and a half for the cornea respectively, the in vivo data agreed with the simulated profile without contradiction. The drug concentration gradient simulated in the vitreous body near the RCS membrane was more significant in the inflamed eyes than in the normal eyes, suggesting that the elimination of the drugs from the RCS membrane was enhanced by inflammation. The present diffusion model can better describe the ocular pharmacokinetics in both normal and diseased conditions.

Key words ocular pharmacokinetics; diffusion; topical; inflammation; ciprofloxacin; ofloxacin

It is well known that ocular bioavailability after topical instillation is extremely low compared with the other administration including oral administration, transdermal application, vitreous injection, and implantable delivery. The ocular bioavailability is also affected by a variety of pharmaceutical factors such as formulation viscosity and the particle size of suspension, and so on. In spite of the fact that the elimination of the drugs from the eye is influenced by the pathological conditions of the barrier membranes such as the retina/choroid/sclera (RCS) membrane and cornea, little is known with respect to the effect of pathological conditions on the ocular pharmacokinetics. Drugs are usually administered under diseased conditions that may differ from those present normally. It is therefore difficult to predict drug movement in the eye under diseased conditions with conventional compartment models assuming uniform concentrations in each ocular tissue. ${ }^{1)}$

In the present study, we have developed the pharmacokinetic model for ocular drug delivery on the basis of the diffusion model. ${ }^{2}$ The concentration profiles after topical instillation calculated were compared with the in vivo rabbit experimental data reported in the literature. In addition, we have examined drug movement in the eye with pathology by assuming a barrier capacity of the surrounding membranes decreased under pathological conditions.

\footnotetext{
Experimental

Öztürk et al. have performed the following in vivo experiments ${ }^{3,4}$ and obtained the result of the pharmacokinetic difference between the disease condition and normal condition. In this study, we used as is in vivo experiments of Öztürk et al. and compared with the result simulated.

In Vivo Experiments of Öztürk et al. ${ }^{3,4)}$ Inflammation was achieved on the right eyes of New Zealand albino rabbits. After the ocular trauma was made with blade and then repaired, intraocular infection was induced by intravitreal injection of a suspension of Staphylococcus aureus. ${ }^{5,6)}$ The intact left eye was used as control. Twenty-four hours after $S$. aureus inoculation, $0.3 \%$ ciprofloxacin or $0.3 \%$ ofloxacin were administered by topical instillation to each eye of normal and inflamed rabbits every hour for 7 and $14 \mathrm{~h}$. Samples were obtained at $30 \mathrm{~min}$ after the last instillation and each drug concentration in aqueous and vitreous humors was then measured using high-pressure liquid chromatography analysis.
}

* To whom correspondence should be addressed.

e-mail: kayoko-ueda@senju.co.jp

In Silico Study A pharmacokinetic model, a spherical and modified cylindrical eye model, was developed as shown in Fig. $1 .^{2)}$ In this pharmacokinetic model, the drug is assumed to be eliminated across three different diffusion pathways: the anterior chamber surface, posterior chamber surface, and vitreous body surface (Fig. 1). ${ }^{2}$

The drug concentration in each eye tissue is evaluated by the equation $\mathrm{Eq}$ 1 assuming neither binding nor metabolism in the eye. ${ }^{2}$

$$
\frac{\partial C}{\partial t}=\frac{1}{x} \frac{\partial}{\partial x}\left(x D \frac{\partial C}{\partial x}\right)+\frac{\partial}{\partial y}\left(D \frac{\partial C}{\partial y}\right)
$$

where $D$ is the diffusion coefficient.

The diffusion coefficient $D$ was determined from the previous in vitro penetration experiments (Table 1). . $^{2,7,8)}$

The appropriate initial and boundary conditions are described in Eqs. 2 to 7.2)

$$
\begin{aligned}
t>0, \quad x=0 ; \quad \frac{d C}{d x} & =0 \\
t>0, \quad x=R ; \quad \frac{d C}{d x} & =\frac{D_{\mathrm{a}}}{D} \frac{K_{\mathrm{a}}}{L_{\mathrm{a}}}\left(C-C_{\mathrm{a}}\right) \quad(1.6<y / R<2.0) \\
\frac{d C}{d x} & =\frac{D_{\mathrm{p}}}{D} \frac{K_{\mathrm{p}}}{L_{\mathrm{p}}}\left(C-C_{\mathrm{p}}\right) \quad(1.5<y / R \leqq 1.6)
\end{aligned}
$$

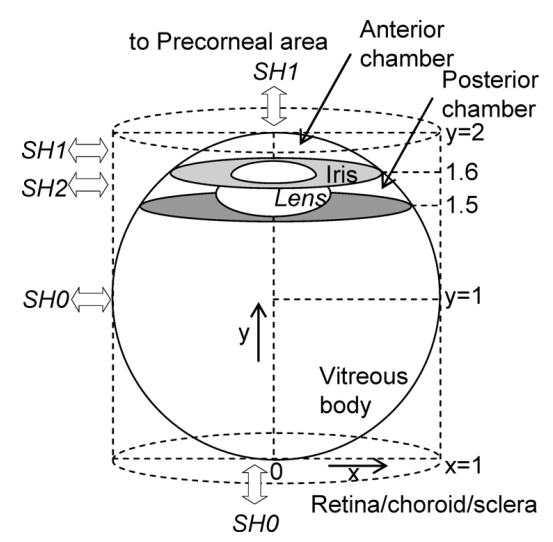

Fig. 1. A Spherical and Modified Cylindrical Eye Model for Pharmacokinetic Evaluation of Ocular Drug Delivery 


$$
\begin{array}{r}
\frac{d C}{d x}=\frac{D_{\mathrm{r}}}{D} \frac{K_{\mathrm{r}}}{L_{\mathrm{r}}}\left(C-C_{\mathrm{r}}\right) \quad(0<y / R \leqq 1.5) \\
t>0, \quad y=0 ; \quad \frac{d C}{d y}=\frac{D_{\mathrm{r}}}{D} \frac{K_{\mathrm{r}}}{L_{\mathrm{r}}}\left(C-C_{\mathrm{r}}\right) \\
y=H ; \quad \frac{d C}{d y}=\frac{D_{\mathrm{a}}}{D} \frac{K_{\mathrm{a}}}{L_{\mathrm{a}}}\left(C-C_{\mathrm{a}}\right)
\end{array}
$$

where $R$ and $H$ are the effective radius and height of eyeball, $D$ is the diffusion coefficient in the eye $\left[\mathrm{cm}^{2} / \mathrm{s}\right], K$ is the membrane partition coefficient, $L$ is the membrane thickness, the subscripts, a, p, r refer to the anterior chamber membrane, the posterior chamber membrane and RCS membrane, respectively.

The rate of drug movement can be characterized by the Sherwood number defined as follows ${ }^{2}$ :

$$
S H=\frac{D m}{D} \frac{K R}{L}
$$

where $D m$ is the diffusion coefficient in each eye tissue $\left[\mathrm{cm}^{2} / \mathrm{s}\right]$.

Model parameters such as the diffusion coefficient and partition coefficient in the cornea for solving Eq. 8 were determined from the bi-layer membrane model according to the each compound property. ${ }^{9)}$ The diffusion coefficients were evaluated from empirical correlation equations in terms of the diffusion coefficient and the molecular weight based on the model compound pirenoxine (Eqs. 9, 10). ${ }^{10)}$

$$
\begin{aligned}
& D e p=D e p A \times\left(\frac{1}{(M \cdot W \cdot / M \cdot W \cdot A .)^{3.0}}\right) \\
& D s t=D s t A \times\left(\frac{1}{(M . W . / M \cdot W \cdot A .)^{0.5}}\right)
\end{aligned}
$$

where Dep is the diffusion coefficient across the epithelium $\left[\mathrm{cm}^{2} / \mathrm{s}\right]$, Dst is the diffusion coefficient across the stroma $\left[\mathrm{cm}^{2} / \mathrm{s}\right], \operatorname{Dep} A$ is the diffusion co-

\begin{tabular}{|c|c|}
\hline & $\left(\mathrm{cm}^{2} / \mathrm{s}\right)$ \\
\hline Vitreous body, $D v^{2,7)}$ & $1.0 \times 10^{-5}$ \\
\hline Anterior chamber ${ }^{2)}$ & $D v \times 10$ \\
\hline Posterior chamber $\left.{ }^{2}\right)$ & $D v \times 10$ \\
\hline Lens capsule 2,8$)$ & $D v / 100$ \\
\hline Lens cortex ${ }^{2,8)}$ & $D v / 100$ \\
\hline Lens nucleus ${ }^{2,8)}$ & $D v / 250$ \\
\hline Iris body ${ }^{2}$ & $D v / 2$ \\
\hline Outside of inscribed sphere ${ }^{2)}$ & $D v \times 1000$ \\
\hline
\end{tabular}
efficient of pirenoxine across the epithelium $\left[6.6 \times 10^{-9} \mathrm{~cm}^{2} / \mathrm{s}^{11)}\right]$, DstA is the diffusion coefficient of pirenoxine across the stroma $\left[2.6 \times 10^{-7} \mathrm{~cm}^{2} / \mathrm{s}^{11)}\right]$, and M.W.A. is the molecular weight of pirenoxine (308).

It was found by Maurice that the diffusion coefficient of the cornea is

Table 1. Diffusion Coefficient in Each Eye Tissues

The diffusion coefficients of vitreous body and lens tissues were determined from in vitro experiments. The other was assumed on the basis of the diffusion coefficient of vitreous body, $D v$. mainly affected by the molecular weight of the penetrant. ${ }^{1,12)}$ Since the molecular weights of ciprofloxacin and ofloxacin are similar to pirenoxine, as used in this study, the diffusion coefficients across the epithelium and stroma were almost equal with those of pirenoxine. ${ }^{11)}$ The diffusion coefficient in the eye is not constant but varies markedly in the ocular tissues as shown in Table 1. The diffusion coefficient in the lens was extremely low and gives rise to a slow release reservoir function. Sherwood numbers, which characterize the barrier capacity of the surrounding tissues, were evaluated by assuming the pseudo-steady state approach (PSSA). ${ }^{2)}$

The partition coefficient could be evaluated from the correlation equations in terms of the octanol/buffer partition coefficient previously determined by various model compounds including beta-adrenergic blocking agents, steroids, and aldose reductase inhibitors (Eqs. 11, 12). ${ }^{13}$ )

$$
\begin{aligned}
& K(\text { epithelium } / \text { buffer })=18.19 \times K(\text { octanol/buffer })^{0.793} \\
& K(\text { stroma/buffer })=4.16 \times K(\text { octanol } / \text { buffer })^{0.125}
\end{aligned}
$$

The membrane thickness in each ocular tissue for solving the Sherwood number was evaluated as the dimensionless ratio of actual membrane thickness to the spherical radius.

The model parameters are shown in Table 2. In the inflamed eye, the barrier capacity of surrounding membrane, the RCS membrane and the cornea, was assumed to decrease as a result of inflammation. In this study the initial drug concentration on the surface of the cornea was $45 \%$ of the formulation concentration based on the instantaneous dilution by tear fluid. ${ }^{14,15)}$

Ciprofloxacin and ofloxacin, suggested as agents in the treatment and prevention of endophthalmitis, ${ }^{16,17)}$ were used as model drugs in this pharmacokinetic simulation study.

The elimination half-life in human tear may reach 20 min under normal blinking conditions ${ }^{1)}$ although the detailed elimination rate was not elucidated yet. The half-life in the tear fluid increases considerably in the rabbit eye since the blinking rate in the rabbit is much smaller than in human eyes. The present rabbit study, therefore, the elimination half-life was assumed approximately two hours; correspondingly the elimination rate of $1 \times 10^{-4}$ $(1 / \mathrm{s})$.

\section{Results}

Simulation of Drug Concentration in Normal Eye By applying the model parameters listed in Table 2, the concentrations in ocular tissues were simulated for the normal eyes of rabbits after single instillation. The simulated concentrations for aqueous and vitreous humors are shown in Figs. 2a and c. Not only an initial distribution period but a late absorption period is also observed along with an elimination period after peak concentration.

The pharmacokinetics for repeated administration was cal-

\begin{tabular}{|c|c|c|c|c|}
\hline & \multicolumn{2}{|c|}{ Ciprofloxacin } & \multicolumn{2}{|c|}{ Ofloxacin } \\
\hline & Normal & Inflamed & Normal & Inflamed \\
\hline Molecular weight & \multicolumn{2}{|c|}{331.35} & \multicolumn{2}{|c|}{361.37} \\
\hline Partition coefficient & \multicolumn{2}{|c|}{$0.056^{24)}$} & \multicolumn{2}{|c|}{$0.33^{25)}$} \\
\hline SH0 (Vitreous) & $1.55 \times 10^{-4}$ & $1.55 \times 10^{-3}$ & $6.04 \times 10^{-4}$ & $6.04 \times 10^{-3}$ \\
\hline SH1 (Tear) & $3.27 \times 10^{-6}$ & $6.54 \times 10^{-6}$ & $1.03 \times 10^{-5}$ & $2.06 \times 10^{-5}$ \\
\hline SH2 (Post chamber) & \multicolumn{2}{|c|}{$1.45 \times 10^{-1}$} & \multicolumn{2}{|c|}{$1.81 \times 10$} \\
\hline Rate of elimination in tears $(1 / \mathrm{s})$ & \multicolumn{2}{|c|}{$1.0 \times 10^{-4}$} & \multicolumn{2}{|c|}{$1.0 \times 10^{-4}$} \\
\hline Anterior concentration $(\mu \mathrm{g} / \mathrm{ml})$ & \multicolumn{2}{|c|}{1350} & \multicolumn{2}{|c|}{1350} \\
\hline
\end{tabular}
culated on the basis of single doses. We found a good agreement between simulated profiles and in vivo experiments by Öztürk as shown in Figs. 2b and d.

Simulation of Drug Concentrations in Inflamed Eye Table 3 compares the aqueous and vitreous humors concentrations between the in vivo experiments and the present in

Table 2. Model Parameters

Each SH was evaluated from the diffusion and partition coefficients of the tissue. The rate of elimination in tear was assumed considering the compound property. Anterior concentrations were calculated from drug formulation concentration $(0.3 \%)$ assuming a $45 \%$ rate of dilution by tears. 
a)

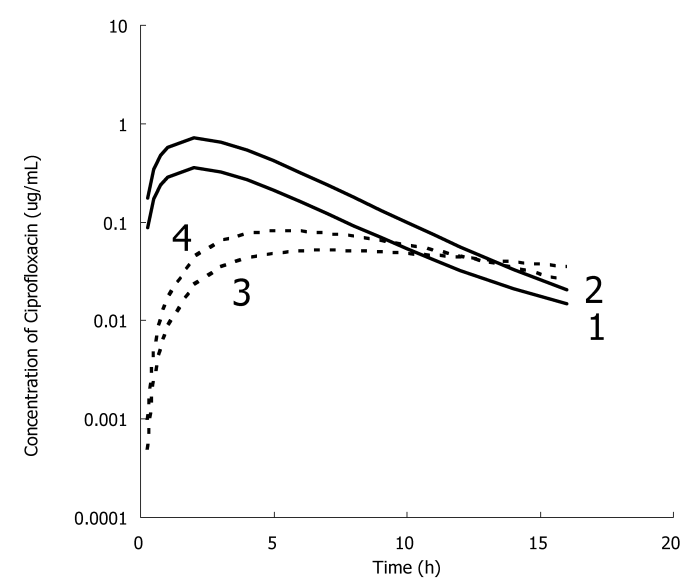

c)

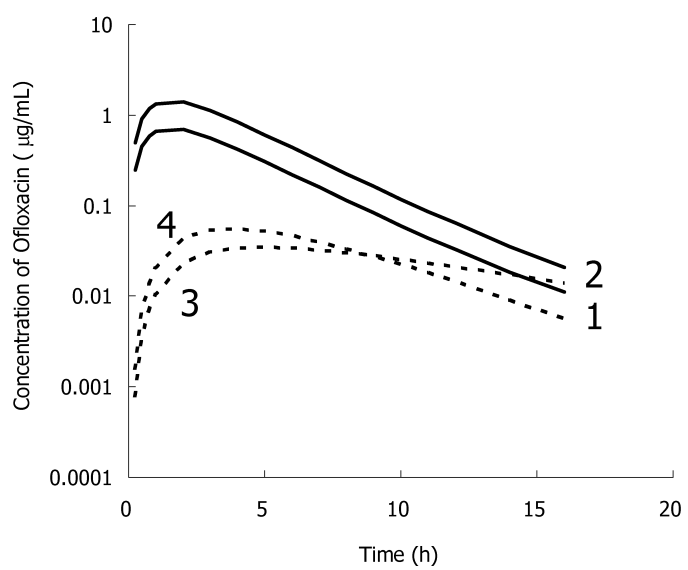

b)

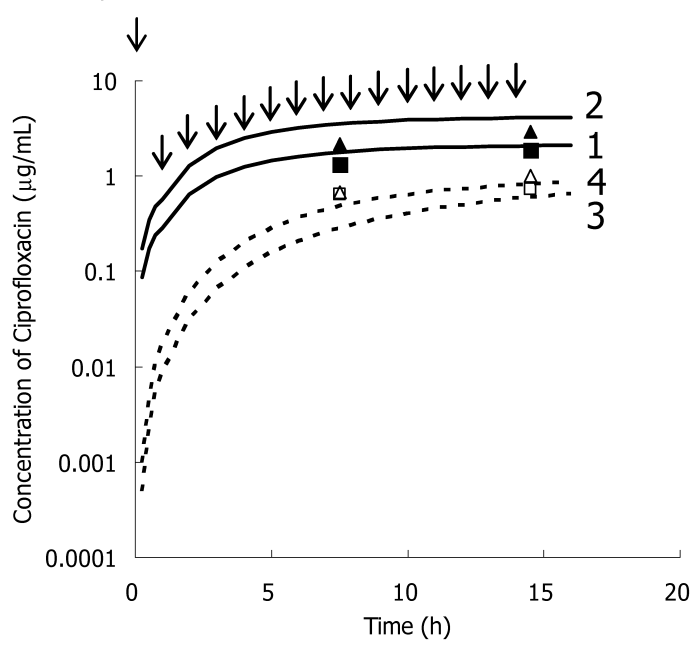

d)

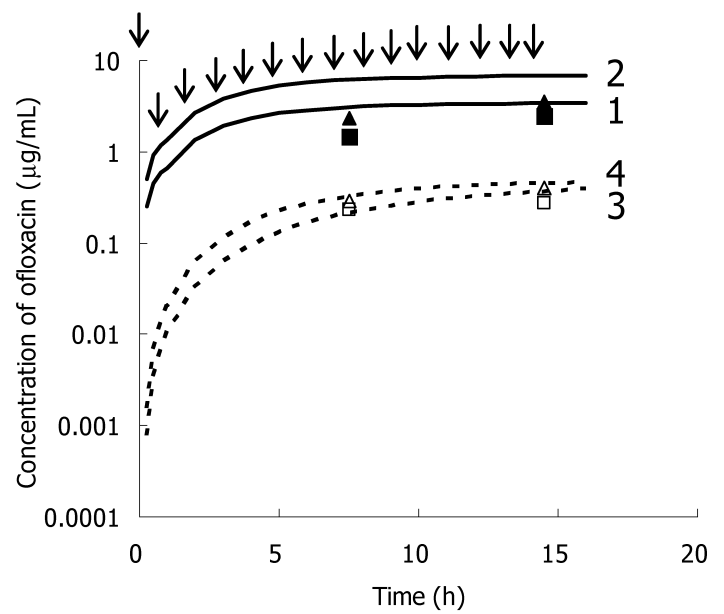

Fig. 2. Simulated Time Course of Concentrations of Ciprofloxacin and Ofloxacin in Aqueous and Vitreous Humors of Normal and Inflamed Eyes

a) Ciprofloxacin (single dose), b) ciprofloxacin (repeated dose), c) ofloxacin (single dose), d) ofloxacin (repeated dose), line 1: calculated aqueous humor (normal), line 2: calculated aqueous humor (inflamed), dashed line 3: calculated vitreous humor (normal), dashed line 4: calculated vitreous humor (inflamed), $\mathbf{a}$ : experimental aqueous humor (normal) $\left(\right.$ Öztürk et $a l^{3,4)}$ ), $\mathbf{\Delta}$ : experimental aqueous humor (inflamed) (Öztürk et al. ${ }^{3,4)}$ ), $\square$ : experimental vitreous humor (normal) (Öztürk et al. ${ }^{3,4)}$ ), $\triangle$ : experimental vitreous humor (inflamed) (Öztürk et al. ${ }^{3,4)}$ ), arrow: administration time.

Table 3. A Comparison between in Vivo Experiments and in Silico Study $(\mu \mathrm{g} / \mathrm{ml})$

\begin{tabular}{|c|c|c|c|c|c|}
\hline \multirow{3}{*}{$\begin{array}{l}\text { Ciprofloxacin } \\
\text { In vivo }\end{array}$} & & \multicolumn{2}{|c|}{ Aqueous humor } & \multicolumn{2}{|c|}{ Vitreous humor } \\
\hline & & $7 \mathrm{~h}$ & $14 \mathrm{~h}$ & $7 \mathrm{~h}$ & $14 \mathrm{~h}$ \\
\hline & Normal & $1.31 \pm 0.78$ & $1.85 \pm 1.69$ & $0.65 \pm 0.44$ & $0.72 \pm 0.80$ \\
\hline & Inflamed & $2.18 \pm 1.02$ & $2.91 \pm 2.12$ & $0.67 \pm 0.77$ & $1.01 \pm 0.43$ \\
\hline \multirow{2}{*}{ In silico } & Normal & 1.79 & 2.08 & 0.28 & 0.60 \\
\hline & Inflamed & 3.55 & 4.09 & 0.48 & 0.82 \\
\hline Ofloxacin & & $7 \mathrm{~h}$ & $14 \mathrm{~h}$ & $7 \mathrm{~h}$ & $14 \mathrm{~h}$ \\
\hline \multirow[t]{2}{*}{ In vivo } & Normal & $1.45 \pm 0.93$ & $2.48 \pm 0.33$ & $0.23 \pm 0.14$ & $0.27 \pm 0.10$ \\
\hline & Inflamed & $2.35 \pm 1.84$ & $3.49 \pm 1.47$ & $0.29 \pm 0.11$ & $0.40 \pm 0.09$ \\
\hline \multirow[t]{2}{*}{ In silico } & Normal & 3.08 & 3.41 & 0.21 & 0.37 \\
\hline & Inflamed & 6.14 & 6.78 & 0.32 & 0.45 \\
\hline
\end{tabular}

Concentrations are mean \pm standard deviation

silico evaluations. The values of the model parameters are shown in Table 2. After trying a variety of combinations for SH0 (Vitreous) and SH1 (Tear), the in vivo data ${ }^{3,4)}$ were explained without contradiction when SH0 and SH1 were 10 times and twice increased comparing to the normal conditions, respectively.
Figure 3 shows the simulated time profiles of ciprofloxacin concentration in the normal eye Figs. $3 \mathrm{a}-\mathrm{d}$ and in the inflamed eye Figs. $3 \mathrm{e}-\mathrm{h}$ after single instillation. The drug diffuses from the tear fluid to the posterior vitreous body through the posterior aqueous humor initially Figs. $3 a$ and $b$. The lens, however, acts as an important reservoir in the late 
a)

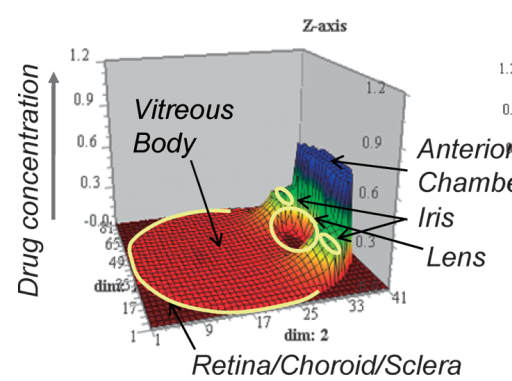

e)

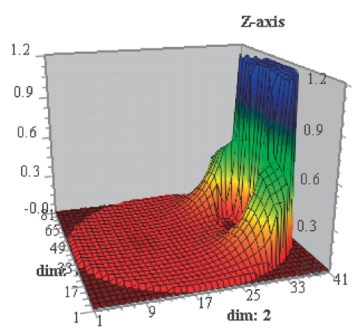

b)

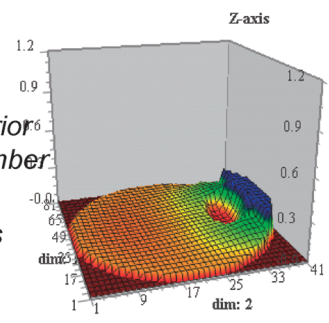

f)

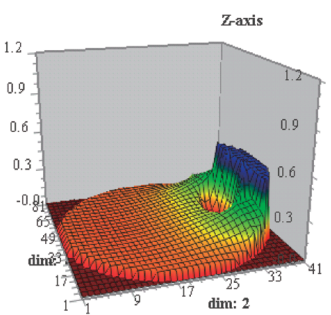

c)

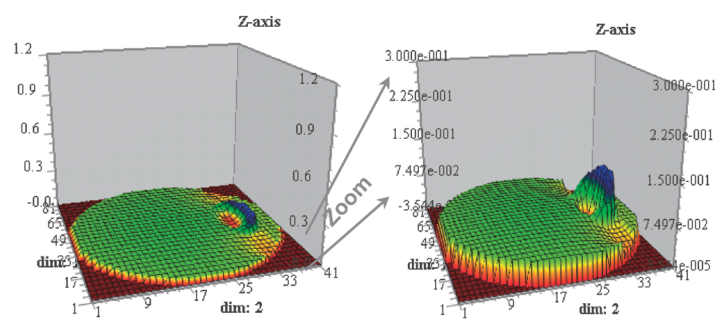

h)

Fig. 3. Ciprofloxacin Concentration Profiles in Eyes after Topical, Single Instillation

a) Normal, $2 \mathrm{~h}, \mathrm{~b}$ ) normal, $6 \mathrm{~h}, \mathrm{c})$ normal, $12 \mathrm{~h}, \mathrm{~d}$ ) normal, $12 \mathrm{~h}, \mathrm{e}$ ) inflamed, $2 \mathrm{~h}, \mathrm{f}$ ) inflamed, $6 \mathrm{~h}, \mathrm{~g}$ ) inflamed, $12 \mathrm{~h}, \mathrm{~h}$ ) inflamed, $12 \mathrm{~h}$.

period. After approximately $12 \mathrm{~h}$, the lens concentration reaches the peak concentration and thereafter the lens play a critical role for ocular reservoir function. The trends of the concentration-time profile agree with the experimental findings by Kinsey and Maurice. ${ }^{18)}$

The highest concentration in the aqueous humor was achieved around two hours after topical instillation in both normal and inflamed eyes. After the drug had been transferred posteriorly, they reached the vitreous body. The drug molecules were then eliminated from the RCS membrane around $6 \mathrm{~h}$ after instillation. In the vitreous humor of normal eyes, drugs were uniformly distributed throughout the vitreous body (Fig. 3d). On the other hand, marked concentration gradients appeared in the inflamed eyes, indicating the significant decrease in the barrier capacity in the surrounding membranes by inflammation (Fig. $3 \mathrm{~h}$ ). Similar profiles were obtained for ofloxacin.

\section{Discussion}

The in vivo data obtained by Öztürk et $a l^{3,4)}$ were compared with the simulated profiles by the present pharmacokinetic model. ${ }^{2}$ ) When blood-retinal barrier was reduced by vascular endothelial growth factor (VEGF), dye leaked more from retina than that without VEGF by $\mathrm{Xu}$ et $a{ }^{19}{ }^{19)}$ and Miyamoto $^{20)}$ et al. For the present simulation, the drug concentration in the inflamed eyes has been analyzed by assuming the reduced barrier capacity of the surrounding membrane, such as the RCS membrane and the cornea. The barrier capacity of the surrounding membrane is inversely related by the Sherwood number (Eq. 8). As mentioned above, the simulated profiles by using a various combination of SHO and SH1 were compared with the experimental findings by Öztürk et al. ${ }^{3,4)}$ We found that the experimental concentrations agreed with the simulated profiles when the values of SH0 and SH1 were increased by inflammation 10-fold and twice of those under normal conditions, respectively. Because the barrier capacity depends on the severity of inflammation, the present values of the Sherwood numbers may be applied only to the present experimental conditions. However Maurice reported the corneal endothelial permeability of $\mathrm{Na}$ ion was two to three times increased by mild trauma under in vivo rabbit experiments. ${ }^{21)}$ In the in vivo experiments, inflammation was also induced by intravitreous injection of a suspension of Staphylococcus aureus. Ten times increase of SH0 for the RCS membrane may be caused partly by the active transport that may occur in the retina. This can attribute to the barrier capacity for the RCS membrane much higher than that in the corneal membrane.

This study focused on the permeability and diffusion of drugs; the convective vitreous flow was not considered though it might play a role in ocular pharmacokinetics. The literature suggests that the vitreous convective flow is higher in diseased than in normal conditions. ${ }^{22)}$

Ocular pharmacokinetics may depend on the compound. Drug concentrations in the vitreous humor of inflamed eyes at $10 \mathrm{~h}$ after administration was equal to or lower than in the normal eye. In addition, ciprofloxacin was eliminated more slowly than ofloxacin from the inflamed vitreous body (Figs. $2 \mathrm{a}, \mathrm{c})$. According to Pitkänen et al., movement of more lipophilic compounds was more appreciable from the vitreous toward the sclera. The retinal pigment epithelium has been found to be a barrier in the retinal delivery of hydrophilic drugs. ${ }^{23)}$ Consequently, the hydrophobic RCS membrane functions as the barrier against ciprofloxacin, which is more hydrophilic than ofloxacin, even under inflammation.

\section{Conclusion}

A diffusion model assuming a modified cylindrical eye has been developed for the ocular pharmacokinetics under normal and inflamed eyes. The inflamed eyes were characterized 
by decreasing the barrier capacity of the surrounding eye tissues such as the RCS membrane, the cornea. The barrier capacity was also characterized by the Sherwood number derived by the pseudo-steady state approach on the boundary membrane. In the present inflammation, the barrier capacity under inflamed conditions was approximately one-tenth and half for the RCS membrane and the cornea, respectively.

Pharmacokinetic experiments are usually performed under normal condition. However, the drugs are clinically administered under diseased conditions. The diffusion model can be used to analyze the drug movement in the variety of pathological ocular tissues. The present in silico approach significantly reduce the need for animals and clinical trials.

\section{References}

1) Maurice D. M., Mishima S., "Pharmacology of the Eye," ed. by Sears M. L., Springer, Berlin, 1984, pp. 19-116.

2) Tojo K., Chem. Pharm. Bull., 52, 1290-1294 (2004).

3) Öztürk F., Kurt E., İnan Ü. Ü., Kortunay S., İlker S. S., Bașci N. E., Bozkurt A., Int. J. Pharm., 204, 97-100 (2000).

4) Öztürk F., Kortunay S., Kurt E., İnan Ü. Ü., İlker S. S., Bașci N., Bozkurt A., Curr. Eye Res., 19, 461-464 (1999).

5) Cleary P. E., Ryan S. J., Am. J. Ophthalmol., 88, 212-220 (1979).

6) Öztürk F., Kortunay S., Kurt E., İlker S. S., İnan Ü. Ü., Başci N. E., Bozkurt A., Kayaalp O., Retina, 19, 127-130 (1999).

7) Uno K., Ohtori A., Tojo K., J. Eye (Atarashii Ganka), 11, 607-609
(1994).

8) Tojo K., J. Jpn. Soc. Cataract. Res., 13, 5-12 (2001).

9) Tojo K., Chiang C. C., Chien Y. W., J. Pharm. Sci., 76, 123-126 (1987).

10) Tojo K., $D N \& P ., 3,409-416$ (1990).

11) Tojo K., Desai D. S., J. Eye (Atarashii Ganka), 8, 945-948 (1991).

12) Maurice D. M., Am. J. Ophthalmol., 47, 361-368 (1959).

13) Tojo K., Ohtori A., Proc. Int. Symp. Control. Rel. Bioact. Mater, 17, 301-302 (1990).

14) Adler C. A., Maurice D. M., Paterson M. E., Exp. Eye Res., 11, 34 42 (1971).

15) Sugaya M., Nagataki S., Jpn. J. Ohthlmol., 22, 127-141 (1978).

16) Neu H. C., Am. J. Ophthalmol,. 112, 15S-24S (1991).

17) Pavesio C., Ophthalmology, 104, 1902-1909 (1997).

18) Kinsey V. E., Maurice D. M., Exp. Eye Res., 3, 156-165 (1964).

19) Xu Q., Qaum T., Adamis A. P., Invest. Ophthalmol. Vis. Sci., 42, 789 794 (2001).

20) Miyamoto K., Khosrof S., Bursell S. E., Moromizato Y., Aiello L. P., Ogura Y., Adamis A. P., Am. J. Pathol., 156, 1733-1739 (2000).

21) Maurice D. M., J. Physiol., 112, 367-391 (1951).

22) Krishnamoorthy M. K., Park J., Augsburger J. J., Banerjee R. K., J. Ocul. Pharmacol. Ther, 24, 255-267 (2008).

23) Pitkänen L., Ranta V. P., Moilanen H., Urtti A., Invest. Ophthalmol. Vis. Sci., 46, 641-646 (2005).

24) Mehri M. H., Yves X. F., Jean-Claude P., Antimicrob. Agents Chemother, 35, 2091-2097 (1991).

25) Hirai K., Aoyama H., Irikura T., Iyobe S., Mitsuhashi S., Antimicrob. Agents Chemother., 29, 535-538 (1986). 